\section{Will gemcitabine monotherapy be dethroned as the adjuvant chemotherapy in pancreatic adenocarcinoma?}
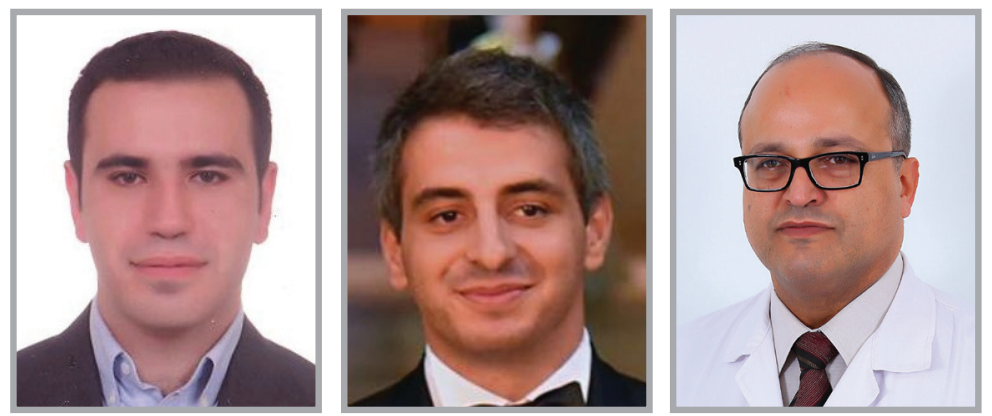

Hampig Raphael Kourie ${ }^{*}, 2$, Joseph Gharios ${ }^{3} \&$ Joseph Kattan ${ }^{2}$

First draft submitted: 4 July 2016; Accepted for publication: 30 August 2016; Published online: 14 September 2016

Pancreatic cancer (PC) is one of the most aggressive and belligerent solid tumors. More than $80 \%$ of patients are diagnosed with locally advanced or metastatic disease; wherein only $15-20 \%$ of these cancers beneficiate from frontline surgery for localized malignancies. In all-stage PC, the median overall survival (OS) is less than 6 months, and less than $5 \%$ of patients survive more than 5 years $(2 \%$ in cases of metastatic PC). Moreover, only $30 \%$ of patients with localized disease treated by surgery remain alive for 5 years $[1,2]$. Because of the poor prognosis of this tumor, adjuvant treatment, namely, chemotherapy-based treatment, is unavoidable to enhance the survival outcomes in these patients.

Based on the results of CONKO-001 trial, 6-month gemcitabine as adjuvant treatment for pancreatic adenocarcinoma has been considered the standard of care since 2007 [3]. The long-term outcome of 368 patients after a follow-up of 136 months was published in 2013. It showed that adjuvant gemcitabine for 6 months compared with observation alone resulted in a significant increase in OS (22.8 vs 20.2 months), as well as disease-free survival (13.4 vs 6.7 months) [4]. Moreover, an ESPAC-3 trial comparing 5-fluorouracyl (5-FU)/folinic acid to gemcitabine in the adjuvant setting concluded that 5-FU/folinic acid could be considered as an alternative to gemcitabine, since there was no difference in survival (23 months for 5-FU vs 23.9 months for gemcitabine) [5]. Nevertheless, in Japan, the standard of care for adjuvant chemotherapy in pancreatic adenocarcinoma is S1. This drug compared favorably with gemcitabine in the Japanese population (not Caucasian population) reported a better progressionfree survival (23.2 vs 12.2 months), a better OS (not reached vs 25.9 months), less toxicities and better quality of life [6]. According to ESPAC-1 trial, the adjuvant

\section{KEYWORDS}

- capecitabine - gemcitabine

- pancreatic adenocarcinoma 
“...gemcitabine/ capecitabine combination represents a possible therapeutic option in the adjuvant setting for pancreatic adenocarcinoma, next to gemcitabine single agent.” chemoradiation therapy showed a deleterious effect in the adjuvant setting of pancreatic adenocarcinoma with 10.4 months of recurrencefree survival in the chemoradiation group and 15.2 months in its absence [7].

After almost a decade of gemcitabine use as a single agent in adjuvant PC in western countries, American Society of Clinical Oncology 2016, by the ESPAC-4 trial, was able to present an alternative treatment option, which is the combination of gemcitabine and capecitabine. The combination arm showed a better OS of more than 2 months ( 28 vs 25.5 months). In the ESPAC- 4 trial, one of the largest trials of PC, 732 patients were included; the treatment was started within 12 weeks after the surgery for a planned duration of 24 weeks. The estimated 5 -year survival rates were $28.8 \%$ in the combination arm versus $16.3 \%$ in the gemcitabine $\mathrm{arm}$. It is notable to report that there was a trend to higher the percentage of grade 3/4 adverse events in the combination arm compared with the gemcitabine alone arm $(\mathrm{p}=0.24)[8]$. In the ESPAC-4 trial, the patient characteristics were representative of a real-world PC population. A large proportion of patients had unfavorable prognostic factors, such as locally advanced or aggressive disease, large tumor size or incomplete removal of the tumor. In the CONKO-001 trial, the majority of patients had T3N1 disease prior to surgery and had undergone an R0 resection.

In parallel, with the positive results of FOLFIRINOX (5-FU, irinotecan and oxaliplatine) in metastatic PC, evaluating this chemotherapeutical regimen in the adjuvant and neoadjuvant settings became an interesting issue. Diverse ongoing trials are testing the intensification of chemotherapy in the adjuvant setting by comparing FOLFIRINOX to gemcitabine (NCT02355119), FOLFIRINOX neoadjuvant/adjuvant (perioperative) versus gemcitabine in adjuvant (NCT02172976) and the neoadjuvant versus the adjuvant setting (NCT01314027). Moreover, gemcitabine in combination with nab-paclitaxel, which presented a better OS in the metastatic setting, is also being tested in the adjuvant setting (NCT01964430).

Predictive biomarkers of response for adjuvant chemotherapy in pancreatic adenocarcinoma either for gemcitabine-based or 5-FU-based are being evaluated, as the hENT1 is the most promising predictive biomarker. This is related to the fact that gemcitabine is hydrophilic and therefore passive diffusion through hydrophobic cellular membranes is slow [9]. Permeation through the membranes requires specialized membrane transporters and hENT1 is the most important agent [10]. In a retrospective study evaluating the predictive value of hENT1 expression to gemcitabine or 5-FU adjuvant treatment, microarrays from 434 patients randomized to chemotherapy in the ESPAC-3 trial were stained with the 10D7G2 anti-hENT1 antibody. The results demonstrated that median survival for patients treated with gemcitabine was 17.1 months for those with low hENT1 expression versus 26.2 months for those with high hENT1 expression; while for the 5-FU group, median survival was comparable with 25.6 and 21.9 months for those with low and high hENT1 expressions, respectively. This study concluded that gemcitabine should not be used for patients with low tumor hENT1 expression and recommended a prospective validation of this study [11].

Although gemcitabine/capecitabine is recently considered a possible therapeutic option in the adjuvant treatment of pancreatic adenocarcinoma next to of gemcitabine monotherapy, the alternative strategy of intensification/escalation of adjuvant chemotherapy seems more appealing in this highly incurable disease. Thus, more aggressive combinations such as FOLFIRINOX or gemcitabine/nab-paclitaxel will probably overthrow the combination gemcitabine/capecitabine in the near future. Moreover, it is noteworthy to mention that NRG/RTG 0848 (adjuvant gemcitabine followed by chemoradiation therapy in nonprogressing patients) has recently been amended to allow medical oncologists to use multiagent chemotherapy in the adjuvant setting (RTOG, clinical trials).

Moreover, the choice of chemotherapy in the adjuvant setting depends classically on tumor and patient characteristics such as the aggressiveness of the disease (margins and lymph nodes), the age, the comorbidities and the performance status. Based on the patients' characteristics of CONKO-001 and ESPAC-4 trials, the combination of gemcitabine and capecitabine should be most probably reserved to younger patients with good performance status and presenting unfavorable prognostic factors (aggressive disease, large tumor size or incomplete removal of the tumor). More personalized and tailored adjuvant therapy must be looked for by assessing predictive biomarkers. One reliable biomarker could be hENT1 which will select patients who will benefit from gemcitabine-based adjuvant 
chemotherapy compared with 5-FU-based chemotherapy.

In conclusion, gemcitabine/capecitabine combination represents a possible therapeutic option in the adjuvant setting for pancreatic adenocarcinoma, next to gemcitabine single agent. Ongoing adjuvant trials based on more intensive or more personalized treatment will soon compete with this new standard and might overthrow it once more.
Financial \& competing interests disclosure

The authors have no relevant affliations or financial involvement with any organization or entity with a financial interest in or financial conflict with the subject matter or materials discussed in the manuscript. This includes employment, consultancies, honoraria, stock ownership or options, expert testimony, grants or patents received or pending, or royalties.

No writing assistance was utilized in the production of this manuscript.

\section{References}

Papers of special note have been highlighted as:

- of interest; $\bullet$ of considerable interest

1 Jemal A, Bray F, Center MM, Ferlay J, Ward E, Forman D. Global cancer statistics. $C A$ Cancer J. Clin. 61, 69-90 (2011).

2 Cameron JL, Riall TS, Coleman J, Belcher KA. One thousand consecutive pancreaticoduodenectomies. Ann. Surg. 244(1), 10-15 (2006).

3 Oettle H, Post S, Neuhaus P et al. Adjuvant chemotherapy with gemcitabine vs observation in patients undergoing curativeintent resection of pancreatic cancer: a randomized controlled trial. JAMA 297(3), 267-277 (2007).

4 Oettle H, Neuhaus P, Hochhaus A et al. Adjuvant chemotherapy with gemcitabine and long-term outcomes among patients with resected pancreatic cancer: the CONKO-001 randomized trial. JAMA 310(14), 1473-1481 (2013).

- An important trial confirming the results of the pivotal trial leading to the approval of gemcitabine in the adjuvant setting in the pancreatic cancer.
5 Neoptolemos JP, Stocken DD, Bassi C et al. Adjuvant chemotherapy with fluorouracil plus folinic acid vs gemcitabine following pancreatic cancer resection: a randomized controlled trial. JAMA 304(10), 1073-1081 (2010).

- An important trial leading to the approval of gemcitabine in adjuvant setting.

6 Fukutomi A, Uesaka K, Boku N et al. JASPAC 01: randomized Phase III trial of adjuvant chemotherapy with gemcitabine versus $S-1$ for patients with resected pancreatic cancer. J. Clin. Oncol. 31(Suppl.), Abstract 4008 (2013).

-. An important Japanese trial leading to the approval.

7 Neoptolemos JP, Dunn JA, Stocken DD et al. Adjuvant chemoradiotherapy and chemotherapy in resectable pancreatic cancer: a randomised controlled trial. Lancet 358 , 1576-1585 (2001).

8 Neoptolemos JP, Palmer D, Ghaneh P et al. ESPAC-4: a multicenter, international, open-label randomized controlled Phase III trial of adjuvant combination chemotherapy of gemcitabine (GEM) and capecitabine
(CAP) versus monotherapy gemcitabine in patients with resected pancreatic ductal adenocarcinoma. J. Clin. Oncol. 34(Suppl.), Abstract LBA4006 (2016).

-. An important trial rendering the combination of gemcitabine and capecitabine as another possible alternative in the adjuvant pancreatic cancer.

9 Maréchal R, Bachet JB, Mackey JR et al. Levels of gemcitabine transport and metabolism proteins predict survival times of patients treated with gemcitabine for pancreatic adenocarcinoma. Gastroenterology 143, 664-674.e1-e6 (2012).

10 García-Manteiga J, Molina-Arcas M, Casado FJ et al. Nucleoside transporter profiles in human pancreatic cancer cells: role of hCNT1 in 2',2'-difluorodeoxycytidineinduced cytotoxicity. Clin. Cancer Res. 9, 5000-5008 (2003).

11 Greenhalf W, Ghaneh P, Neoptolemos JP et al. Pancreatic cancer hENT1 expression and survival from gemcitabine in patients from the ESPAC-3 trial. J. Natl Cancer Inst. 106(1), djt347 (2014). 